\title{
Quantum-Classical Correspondence in Response Theory
}

\author{
Maksym Kryvohuz and Jianshu Cao* \\ Department of Chemistry, Massachusetts Institute of Technology, Cambridge, Massachusetts 02139, USA
}

(Received 20 April 2005; published 28 October 2005)

The correspondence principle between the quantum commutator $[\hat{A}, \hat{B}]$ and the classical Poisson brackets $\iota \hbar\{A, B\}$ is examined in the context of response theory. The classical response function is obtained as the leading term of the $\hbar$ expansion of the phase space representation of the response function in terms of Weyl-Wigner transformations and is shown to increase without bound at long times as a result of ignoring divergent higher-order contributions. Systematical inclusion of higher-order contributions improves the accuracy of the $\hbar$ expansion at finite times. Resummation of all the higher-order terms establishes the classical-quantum correspondence $\left.\langle v+n|\hat{\alpha}(t)| v\rangle \leftrightarrow \alpha_{n} e^{\text {in } \omega t}\right|_{J_{v}+n \hbar / 2}$. The time interval of the validity of the simple classical limit $[\hat{A}(t), \hat{B}(0)] \rightarrow \iota \hbar\{A(t), B(0)\}$ is estimated for quasiperiodic dynamics and is shown to be inversely proportional to anharmonicity.

DOI: 10.1103/PhysRevLett.95.180405

PACS numbers: 03.65.Sq, 61.20.Lc, 78.30.Cp

Applications of multidimensional spectroscopy to large molecules and condensed phase systems have motivated the calculation of response functions using classical dynamics.[1-7]. Classical evaluation of response functions usually employs the simple correspondence rule between the quantum commutator $[\hat{A}, \hat{B}]$ and the classical Poisson bracket $\iota \hbar\{A, B\}$. However, the classical response theory has several problems. Van Kampen cautioned the validity of the application of classical perturbation theory to the calculation of a system's response [8]. Recent numerical and analytical results demonstrate the divergence of both linear and nonlinear classical response functions at long times $[2,4,9]$. Yet, while the quantum response function is well defined and can be rigorously calculated, the problems appear after the classical limit is taken. The key question is whether the classical limit is taken appropriately. In the present Letter we follow the derivation of the classical limit from the phase space representation of quantum mechanics to show that the simple classical limit of the response function in terms of Poisson brackets is not valid at long times. The upper time limit for the quantumclassical agreement, i.e., the crossover time, is found to be inversely proportional to system's anharmonicity.

The nonlinear response $P^{(n)}(t)$ to the $n$th order in the applied field $E(t)$ is described by

$$
\begin{aligned}
P^{(n)}(t)= & \int_{0}^{\infty} d t_{n} \ldots \int_{0}^{\infty} d t_{1} E\left(t-t_{n}\right) \ldots E\left(t-t_{1}-\ldots t_{n}\right) \\
& \times R^{(n)}\left(t_{n}, \ldots, t_{1}\right),
\end{aligned}
$$

with the $n$th order response function $R^{(n)}$ [10]

$$
\begin{aligned}
R^{(n)}\left(t_{n}, \ldots, t_{1}\right)= & \left(\frac{\iota}{\hbar}\right)^{n} \\
& \times\left\langle\left[\left[\ldots\left[\hat{\alpha}\left(\tau_{n}\right), \hat{\alpha}\left(\tau_{n-1}\right)\right], \ldots, \hat{\alpha}\left(\tau_{1}\right)\right], \hat{\alpha}(0)\right]\right\rangle,
\end{aligned}
$$

where $\tau_{n}=\sum_{i=1}^{n} t_{i}$ and the operator $\hat{\alpha}(\hat{\mathbf{x}}(t), \hat{\mathbf{p}}(t))$ stands for the polarization operator or the dipole operator. The classical expression for the response function is obtained by replacing quantum commutators with Poisson brackets and neglecting the higher-order terms in the Planck constant $(\hbar \rightarrow 0)$

$$
\begin{aligned}
R_{c}^{(n)}\left(t_{n}, \ldots, t_{1}\right)= & (-1)^{n} \\
& \times\left\langle\left\{\left\{\ldots\left\{\alpha\left(\tau_{n}\right), \alpha\left(\tau_{n-1}\right)\right\}, \ldots, \alpha\left(\tau_{1}\right)\right\}, \alpha(0)\right\}\right\rangle,
\end{aligned}
$$

where $\{\ldots\}$ are Poisson brackets. But can we neglect higher-order terms? To answer this question we examine the classical expansion of quantum mechanics. We begin with the Weyl-Wigner-Moyal symbol-calculus approach [11-13], which introduces Weyl transforms (scalar functions) instead of operators and the Wigner function instead of the state vector using the rule

$$
\begin{aligned}
\operatorname{symb}(\hat{A}) & \equiv a_{\hbar}(\mathbf{p}, \mathbf{q}) \\
& =\int d \mathbf{v} e^{(\iota / \hbar) \mathbf{p} \cdot \mathbf{v}}\left\langle\mathbf{q}-\frac{1}{2} \mathbf{v}|\hat{A}| \mathbf{q}+\frac{1}{2} \mathbf{v}\right\rangle
\end{aligned}
$$

The noncommutative Moyal product that corresponds to noncommutative product of quantum operators follows directly from the definition of the Weyl transform

$$
\begin{aligned}
\operatorname{symb}(\hat{A} \hat{B}) & \equiv a_{\hbar} * b_{\hbar} \\
& =a_{\hbar} \exp \left(\frac{\iota \hbar}{2}\left[\frac{\partial}{\partial \mathbf{q}} \frac{\vec{\partial}}{\partial \mathbf{p}}-\frac{\grave{\partial}}{\partial \mathbf{p}} \frac{\vec{\partial}}{\partial \mathbf{q}}\right]\right) b_{\hbar},
\end{aligned}
$$

where the arrows indicate the direction of operation of the derivative. The quantum commutator $[\hat{A}, \hat{B}]$ in the phase space representation then corresponds to Moyal brackets 


$$
\begin{aligned}
\left\{a_{\hbar}, b_{\hbar}\right\}_{\hbar} & =a_{\hbar} * b_{\hbar}-b_{\hbar} * a_{\hbar}=2 \iota a_{\hbar} \sin \left(\frac{\hbar}{2}\left[\frac{\overleftarrow{\partial}}{\partial \mathbf{q}} \frac{\vec{\partial}}{\partial \mathbf{p}}-\frac{\overleftarrow{\partial}}{\partial \mathbf{p}} \frac{\vec{\partial}}{\partial \mathbf{q}}\right]\right) b_{\hbar} \\
& =\iota \hbar\left(\frac{\partial a_{\hbar}}{\partial \mathbf{q}} \frac{\partial b_{\hbar}}{\partial \mathbf{p}}-\frac{\partial a_{\hbar}}{\partial \mathbf{p}} \frac{\partial b_{\hbar}}{\partial \mathbf{q}}\right)-\frac{\iota \hbar^{3}}{24} a_{\hbar}\left(\frac{\overleftarrow{\partial}}{\partial \mathbf{q}} \frac{\vec{\partial}}{\partial \mathbf{p}}-\frac{\overleftarrow{\partial}}{\partial \mathbf{p}} \frac{\vec{\partial}}{\partial \mathbf{q}}\right)^{3} b_{\hbar}+\cdots,
\end{aligned}
$$

where the first term is the classical Poisson brackets multiplied by $\iota \hbar$. Hence, an appropriate phase space representation of the quantum response function takes the form

$$
R^{(n)}\left(t_{n}, \ldots, t_{1}\right)=\left(\frac{\iota}{\hbar}\right)^{n} \int d \mathbf{p} d \mathbf{q} \rho_{\hbar}(\mathbf{p}, \mathbf{q})\left\{\left\{\ldots\left\{\alpha_{\hbar}\left(\tau_{n}\right), \alpha_{\hbar}\left(\tau_{n-1}\right)\right\}_{\hbar}, \ldots, \alpha_{\hbar}\left(\tau_{1}\right)\right\}_{\hbar}, \alpha_{\hbar}(0)\right\}_{\hbar} .
$$

Equations (7) and (2) are equivalent expressions of quantum response functions.

The evaluation of the classical limit of Eq. (7) can be illustrated for quasiperiodic systems. We introduce the semiclassical wave function[14-20] correspondent to eigenvalue $E_{\mathbf{n}}=H\left(\mathbf{J}_{n}=\mathbf{n} \hbar+\boldsymbol{\beta} \hbar\right)$ :

$$
\langle\boldsymbol{\varphi} \mid \mathbf{n}\rangle=(2 \pi)^{-N / 2} e^{\iota \mathbf{n} \varphi},
$$

where $N$ is the dimensionality of the system and $\boldsymbol{\beta}$ is the Maslov index. We use semiclassical wave function (8) to express the Weyl transform in action-angle basis $(\mathbf{J}, \boldsymbol{\varphi})$, [14-17]

$$
\begin{aligned}
a_{\hbar}\left(\mathbf{J}_{n}, \boldsymbol{\varphi}\right) & =\int_{-\pi}^{\pi} d \boldsymbol{\xi} e^{\imath \mathbf{n} \cdot \xi}\left\langle\boldsymbol{\varphi}-\frac{1}{2} \xi|\hat{A}| \boldsymbol{\varphi}+\frac{1}{2} \boldsymbol{\xi}\right\rangle \\
& =\sum_{\mathbf{k}} a_{\mathbf{k}}\left(\mathbf{J}_{n}\right) e^{\imath \mathbf{k} \varphi} .
\end{aligned}
$$

The latter is just a Fourier decomposition of the classical function $a(\mathbf{J}, \boldsymbol{\varphi})=\sum_{\mathbf{k}} a_{\mathbf{k}}(\mathbf{J}) e^{\iota \mathbf{k} \varphi}$. Thus, the Weyl symbols in semiclassical representation (8) are classical functions with quantized actions $\mathbf{J}$. Angle variables $\boldsymbol{\varphi}$ obey classical dynamics as required by WKB approximation (8). The expression (7) thus reads

$$
\begin{aligned}
R_{s}^{(n)}\left(t_{n}, \ldots, t_{1}\right)= & \left(\frac{\iota}{\hbar}\right)^{n} \sum_{\mathbf{k}} \int d \boldsymbol{\varphi} \rho\left(\mathbf{J}_{k}, \boldsymbol{\varphi}\right) \\
& \times\left\{\left\{\ldots\left\{\alpha\left(\tau_{n}\right), \alpha\left(\tau_{n-1}\right)\right\}_{\hbar}, \ldots, \alpha\left(\tau_{1}\right)\right\}_{\hbar}, \alpha(0)\right\}_{\hbar}
\end{aligned}
$$

which differs from the classical expression (3) in the use of the Moyal bracket

$$
\{a, b\}_{\hbar}=2 \iota a \sin \left(\frac{\hbar}{2}\left[\frac{\overleftarrow{\partial}}{\partial \boldsymbol{\varphi}} \frac{\vec{\partial}}{\partial \mathbf{J}}-\frac{\overleftarrow{\partial}}{\partial \mathbf{J}} \frac{\vec{\partial}}{\partial \boldsymbol{\varphi}}\right]\right) b
$$

The classical limit of the Moyal bracket $\{\ldots, \ldots\}_{\hbar}$ follows directly from Eq. (6) by omitting the higher-order terms in the Planck constant and preserving only the first (Poisson-bracket) term: $\lim _{\hbar \rightarrow 0}\{\ldots, \ldots\}_{\hbar}=\iota \hbar\{\ldots, \ldots\}$. Yet, this simple limit is not valid for response theory. The higher-order terms in the expansion (6) can be omitted only if the prefactor of $\hbar^{n}$ is finite. However, this is not the case in response theory. The expression for the response function contains commutators of the same operator $\hat{\alpha}(t)$ evaluated at different times $[\hat{\alpha}(t), \hat{\alpha}(0)]$. The expansion (6) of the Moyal bracket $\{\alpha(t), \alpha(0)\}_{\hbar}$ thus leads to the $n$th order stability matrix $M^{(n)}=\frac{\partial^{n} \mathbf{q}(t)}{\partial \mathbf{q}(0)^{n-k} \partial \mathbf{p}(0)^{k}}$ in each $\hbar^{n}$ term. For the classical motion, stability matrices diverge as $O\left(t^{n}\right)$ for integrable systems and exponentially for chaotic ones. Every $\hbar^{n}$ term in the series of $\{\alpha(t), \alpha(0)\}_{\hbar}$ in Eq. (6) carries a time-divergent factor which becomes infinitely large as $t \rightarrow \infty$, implying that at sufficiently long times a small $\hbar^{n}$ will be compensated by large $t^{n}$. Thus, time divergence of the classical response function arises from the simple limit in the form (3) neglecting terms which can be larger than the leading term at long times. Strictly speaking, taking the usual classical limit $\hbar \rightarrow 0$ we interchange the limits $\lim _{t \rightarrow \infty}$ and $\lim _{\hbar \rightarrow 0}$ which are noncommuting. The noncommutativity of the limits $t \rightarrow \infty$ and $\hbar \rightarrow 0$ was pointed out by Berry in [21]. The response function (7) is well defined for any moment of time, but the exchange of the two limits and the subsequent elimination of higher-order terms of Moyal bracket expansion (6) lead to the wellknown problem of time divergence $[1,3,4,8,9]$. It is worth noting that we do not meet the same difficulties with the limit $\{a, b\}_{\hbar} \rightarrow \iota \hbar\{a, b\}$ (and thus with the correspondence principle $[\hat{A}, \hat{B}] \leftrightarrow \iota \hbar\{a, b\})$ in equilibrium applications where the commutators are evaluated at the same moment of time. Without the presence of stability matrices, the elimination of the higher-order terms in $\hbar$ is justified.

Our main argument is that response functions can be systematically evaluated with classical observables by calculating higher-order terms of Moyal expansion (6). Resummation of the infinite terms in the Moyal bracket expansion converges to a semiclassical result which has oneto-one correspondence to the quantum response function.

We demonstrate the above argument by first establishing the convergence of series for $\left\{a\left(t_{2}\right), b\left(t_{1}\right)\right\}_{\hbar}$ in Eq. (10) for regular systems. Fourier decompositions of $a(t)$ and $b(t)$ reduce expression (11) to

$$
\begin{aligned}
\left\{a\left(t_{2}\right), b\left(t_{1}\right)\right\}_{\hbar}= & \sum_{\mathbf{n}, \mathbf{m}}\left\{e^{(\hbar \mathbf{m} / 2)(\partial / \partial \mathbf{J})}\left(a_{\mathbf{n}} e^{\iota \mathbf{n} \varphi\left(t_{2}\right)}\right)\right\} \\
& \times\left\{e^{-(\hbar \mathbf{n} / 2)(\partial / \partial \mathbf{J})}\left(b_{\mathbf{m}} e^{\iota \mathbf{m} \varphi\left(t_{1}\right)}\right)\right\} \\
& -\sum_{\mathbf{n}, \mathbf{m}}\left\{e^{-(\hbar \mathbf{m} / 2)(\partial / \partial \mathbf{J})}\left(a_{\mathbf{n}} e^{\iota \mathbf{n} \varphi\left(t_{2}\right)}\right)\right\} \\
& \times\left\{e^{(\hbar \mathbf{n} / 2)(\partial / \partial \mathbf{J})}\left(b_{\mathbf{m}} e^{\iota \mathbf{m} \varphi\left(t_{1}\right)}\right)\right\},
\end{aligned}
$$


where $\boldsymbol{\varphi}(t)=\boldsymbol{\omega} t+\boldsymbol{\varphi}_{0}$ with $\boldsymbol{\omega}=\partial E / \partial \mathbf{J}$. Considering the quantum matrix element $\left\langle\mathbf{v}\left|\left[\hat{a}\left(t_{2}\right), \hat{b}\left(t_{1}\right)\right]\right| \mathbf{v}\right\rangle$

$$
\begin{aligned}
\left\langle\mathbf{v}\left|\left[\hat{a}\left(t_{2}\right), \hat{b}\left(t_{1}\right)\right]\right| \mathbf{v}\right\rangle= & \sum_{\mathbf{n}}\left(\left\langle\mathbf{v}\left|\hat{a}\left(t_{2}\right)\right| \mathbf{v}+\mathbf{n}\right\rangle\left\langle\mathbf{v}+\mathbf{n}\left|\hat{b}\left(t_{1}\right)\right| \mathbf{v}\right\rangle\right. \\
& \left.-\left\langle\mathbf{v}\left|\hat{b}\left(t_{1}\right)\right| \mathbf{v}+\mathbf{n}\right\rangle\left\langle\mathbf{v}+\mathbf{n}\left|\hat{a}\left(t_{2}\right)\right| \mathbf{v}\right\rangle\right),
\end{aligned}
$$

it follows from Eq. (12) that its classical correspondence takes the form

$$
\begin{aligned}
\int \sum_{\mathbf{n}}\left\{a\left(t_{2}\right), b\left(t_{1}\right)\right\}_{\hbar} \frac{\delta_{\mathbf{n}, \mathbf{v}}}{(2 \pi)^{N}} d \boldsymbol{\varphi}_{0}= & \sum_{\mathbf{n}}\left(\left.a_{-\mathbf{n}} e^{-\imath \mathbf{n} \boldsymbol{\omega} t_{2}}\right|_{\mathbf{J}=\mathbf{J}_{v}+(\hbar \mathbf{n} / 2)}\right) \\
& \times\left(\left.b_{\mathbf{n}} e^{\imath \mathbf{n} \boldsymbol{\omega} t_{1}}\right|_{\mathbf{J}=\mathbf{J}_{v}+(\hbar \mathbf{n} / 2)}\right) \\
& -\sum_{\mathbf{n}}\left(\left.a_{\mathbf{n}} e^{\iota \mathbf{n} \boldsymbol{\omega} t_{2}}\right|_{\mathbf{J}=\mathbf{J}_{v}+(\hbar \mathbf{n} / 2)}\right) \\
& \left(\left.b_{-\mathbf{n}} e^{-\imath \mathbf{n} \boldsymbol{\omega} t_{1}}\right|_{\mathbf{J}=\mathbf{J}_{v}+(\hbar \mathbf{n} / 2)}\right)
\end{aligned}
$$

which does not lead to a time divergence [in the last step we have used the property of the translational operator $\left.\exp \left(\Delta \frac{\partial}{\partial J}\right) f(J)=f(J+\Delta)\right]$. As a result, the semiclassical response function Eq. (10) maps to the quantum response function (2) through the Heisenberg's correspondence principle $[22,23]$ between the quantum matrix element and classical Fourier component: $\langle v+n|\hat{\alpha}(t)| v\rangle \leftrightarrow$ $\left.\alpha_{n} e^{i n \omega t}\right|_{J_{\nu}+n \hbar / 2}$. We recently used phase space quantization to arrive at the same quantum-classical correspondence and generalized the correspondence principle to nonlinear response functions [3,24]. Surprisingly, the semiclassical expression (10) or equivalently Eq. (14) still leads to exact quantum results for several exactly solvable systems including the harmonic oscillator and Morse oscillator discussed later [3,24].

Let us estimate crossover time when the quantum mechanical effects in the $\hbar$ expansion of Moyal brackets $\{\alpha(t), \alpha(0)\}_{\hbar}$ start to play a significant role. For a 1D system the first two terms of the Moyal bracket expansion (11) are

$$
\begin{aligned}
\{\alpha(t), \alpha(0)\}_{\hbar}= & \iota \hbar\left(\frac{\partial \alpha(t)}{\partial \varphi_{0}} \frac{\partial \alpha(0)}{\partial J}-\frac{\partial \alpha(t)}{\partial J} \frac{\partial \alpha(0)}{\partial \varphi_{0}}\right) \\
& -\frac{\iota \hbar^{3}}{24} \alpha(t)\left(\frac{\overleftarrow{\partial}}{\partial \varphi_{0}} \frac{\vec{\partial}}{\partial J}-\frac{\grave{\partial}}{\partial J} \frac{\vec{\partial}}{\partial \varphi_{0}}\right)^{3} \alpha(0)+\cdots .
\end{aligned}
$$

With Fourier decomposition of $a(t)$ the derivatives $\partial \alpha(t) / \partial J$ in the above expression result in time-divergent terms $\operatorname{t} \iota n \frac{\partial \omega}{\partial J} \alpha_{n} e^{\iota n \omega t+\ln \varphi_{0}}$. At long times, the first term (Poisson-bracket term) in (15) is of the order of the divergent derivative $\left|\frac{\partial \alpha(t)}{\partial J}\right| \sim t\left|\alpha_{\max } \frac{\partial \omega}{\partial J}\right|$; the second is of the order of its highest divergent derivative $\left|\frac{\partial^{3} \alpha(t)}{\partial J^{3}}\right| \sim$ $t^{3}\left|\alpha_{\max }\left(\frac{\partial \omega}{\partial J}\right)^{3}\right|$, where $\left|\alpha_{\max }\right|$ is the largest spectral component. Obviously the second term becomes significant when it is of the same magnitude as the first term $\hbar t\left|\alpha_{\max }\right|^{2} \frac{\partial \omega}{\partial J} \simeq$ $\hbar^{3} t^{3}\left|\alpha_{\max }\right|^{2}\left(\frac{\partial \omega}{\partial J}\right)^{3}$, giving the crossover time

$$
t^{*} \simeq \frac{1}{\hbar\left|\frac{\partial \omega}{\partial J}\right|} .
$$

For the harmonic systems $\partial \omega / \partial J=0$ the crossover time (16) is infinite, implying that the response functions of harmonic systems can be successfully calculated using the single Poisson-brackets term. Equation (16) justifies the known equivalence of quantum and classical response functions for harmonic systems. However, any anharmonicity $\partial \omega / \partial J \neq 0$ leads to the finite value of the crossover time $t^{*}(16)$, and the crossover time decreases with anharmonicity [25]. Beyond the critical time, $t>t^{*}$, one should expect the failure of the correspondence principle $[\hat{\alpha}(t), \hat{\alpha}(0)] \leftrightarrow \iota \hbar\{\alpha(t), \alpha(0)\}$ and thus the need to include higher-order terms in the expansion of the Moyal product.

We illustrate the above arguments with the linear response function of constant-energy Morse oscillator with the Hamiltonian $\hat{H}=-\frac{\hbar^{2}}{2 \mu} \frac{d^{2}}{d q^{2}}+D\left(1-e^{-\sigma q}\right)^{2}$. In Ref. [3], we introduced the one-photon polarization operator $\hat{\alpha}=\left(b+b^{+}\right)$with its classical analog [3] $\alpha(J, \varphi)=$ $2 \sqrt{\frac{\chi_{e}(J / \hbar)^{2}-(J / \hbar)}{1-\chi_{e}}} \cos (\varphi)$, where $\varphi=\left(1-\frac{2 \chi_{e} J}{\hbar}\right) \omega_{0} t+\varphi_{0}$, $\omega_{0}=\sqrt{2 D \sigma^{2} / \mu}$, and $\chi_{e}=\hbar \sigma / \sqrt{8 D \mu}$. The quantum linear response function for a given energy state $E_{v}$ is then

$$
\begin{aligned}
R^{(1)}(t)= & \left(\frac{\iota}{\hbar}\right)\langle v|[\hat{\alpha}(t), \hat{\alpha}(0)]| v\rangle \\
= & \frac{2}{\left(1-\chi_{e}\right) \hbar}\left[v+1-\chi_{e}(v+1)^{2}\right] \\
& \times \sin \left\{\left[1-2 \chi_{e}(v+1)\right] \omega_{0} t\right\}-\frac{2}{\left(1-\chi_{e}\right) \hbar} \\
& \times\left[v-\chi_{e} v^{2}\right] \sin \left\{\left(1-2 \chi_{e} v\right) \omega_{0} t\right\} .
\end{aligned}
$$

The semiclassical expression (10)-(14) gives exactly the same result, when the quantization condition $J_{v}=\hbar(v+$ 1/2) was used. The simple classical limit in Eq. (3) yields

$$
\begin{aligned}
R_{c}^{(1)}(t)= & -\int\{\alpha(t), \alpha(0)\} \frac{\delta\left(J-J_{v}\right)}{2 \pi} d J d \varphi_{0} \\
= & \frac{2}{\left(1-\chi_{e}\right) \hbar}\left[1-\chi_{e}(2 v+1)\right] \\
& \times \sin \left\{\left[1-\chi_{e}(2 v+1)\right] \omega_{0} t\right\}-\frac{\omega_{0} t}{\left(1-\chi_{e}\right) \hbar} \\
& \times\left[1-\left(1-(2 v+1) \chi_{e}\right)^{2}\right] \sin \left\{\left[1-\chi_{e}(2 v+1)\right] \omega_{0} t\right\},
\end{aligned}
$$

which diverges linearly in time. The dependence of the semiclassical result (10) on the number of terms in the Moyal bracket expansion is shown on Fig. 1 for the onedimensional Morse oscillator with parameters $\omega_{0}=5$, $\chi_{e}=0.005, \hbar=1$, and linear polarization operator. The agreement between quantum (2) and classical (3) linear response functions indeed starts to fail after time 

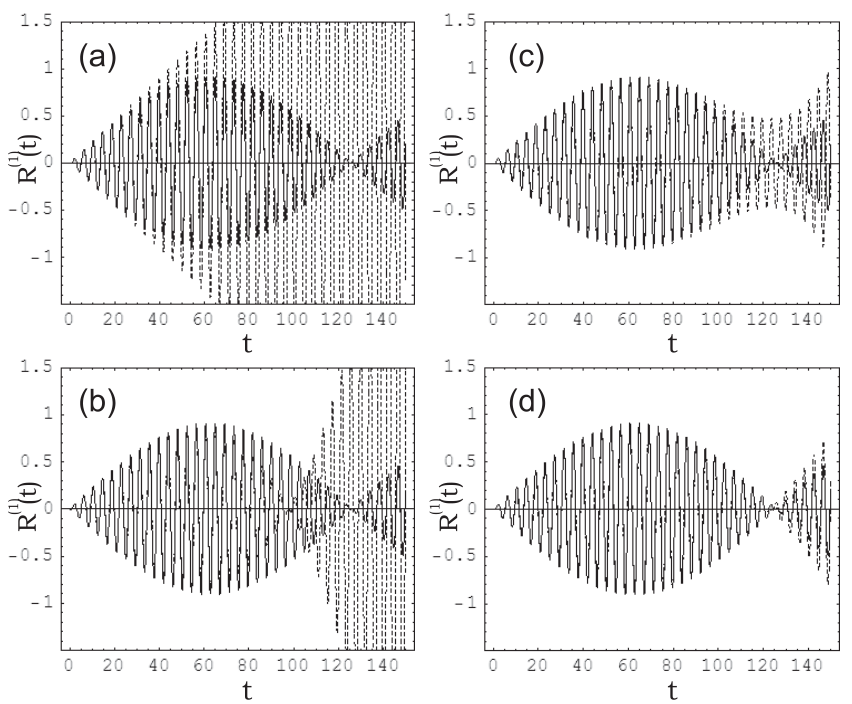

FIG. 1. Linear response functions of constant-energy Morse oscillator. The solid lines represent quantum results from Eq. (2) and the dashed lines represent semiclassical result from Eq. (10) for the case of: (a) single Poisson-bracket term in the expansion of Moyal bracket; (b) two lowest order terms of the expansion ( $\hbar$ term and $\hbar^{3}$ term); (c) three lowest order terms ( $\hbar, \hbar^{3}, \hbar^{5}$ terms); (d) four lowest terms $\left(\hbar, \hbar^{3}, \hbar^{5}, \hbar^{7}\right.$ terms $)$.

$t^{*}=1 /\left(\hbar\left|\frac{\partial \omega}{\partial J}\right|\right)=1 /\left(2 \chi_{e} \omega_{0}\right)=20$ [Fig. 1(a)]. As we systematically include higher-order terms of Moyal bracket expansion the agreement with the quantum result extends to longer times. The account of all terms of Moyal bracket expansion gives the exact quantum result.

Let us estimate the crossover time $t^{*}$ for real systems, liquids $C S_{2}$, and $\mathrm{Xe}$. The curvature of Morse potential $V_{M}(r)=\left(\epsilon\left(e^{\sigma\left(1-r / r_{e}\right)}-1\right)^{2}-\epsilon\right)$ coincides well with Lennard-Jones potential $V_{L J}=4 \epsilon\left((\xi / r)^{12}-(\xi / r)^{6}\right)$ for $\sigma=6$ with $r_{e}=\sqrt[6]{2} \xi$ [26]. For $C S_{2}$ molecule with $\mu=$ 76 a.u. and mean Lennard-Jones radius $\xi \simeq 3.5 \AA$ we have $t^{*}=1 / 2 \chi_{e} \omega_{0}=\mu(\sqrt[6]{2} \xi)^{2} / \hbar \sigma^{2} \simeq 5$ ps. For $X e, \mu=$ 131 a.u. and $\xi=3.91 \AA$, thus $t^{*} \simeq 10$ ps. Both times are on the same order of the time scales of the reported experiments and MD simulations [5,7]. However, MD simulations of real systems do not observe the divergence of the response functions. It was demonstrated in [27] that the response functions for irregular dynamics may converge. Research is being continued to find the quantum-classical correspondence for chaotic and dissipative systems. Yet, the crossover time $t^{*}$ derived in this Letter remains a good estimation for the time interval of the validity of the classical approximation to the exact quantum results in response theory.

We have shown that the problem of the time divergence of the classical response function stems from the interchange of noncommuting limits $\hbar \rightarrow 0$ and $t \rightarrow \infty$, which results in the elimination of the higher-order terms of the
Weyl transform of quantum commutator $[\hat{A}(t), \hat{B}(0)]$. The proposed semiclassical expression (10) removes the classical divergence. The accuracy of classical dynamics can be systematically improved by incorporating higher-order corrections beyond the crossover time.

*Email address: jianshu@mit.edu

[1] J. A. Leegwater and S. Mukamel, J. Chem. Phys. 102, 2365 (1995).

[2] S. Mukamel, V. Khidekel, and V. Chernyak, Phys. Rev. E 53, R1 (1996).

[3] J. Wu and J. Cao, J. Chem. Phys. 115, 5381 (2001).

[4] W. G. Noid, G. S. Ezra, and R. F. Loring, J. Phys. Chem. B 108, 6536 (2004).

[5] A. Ma and R. M. Stratt, Phys. Rev. Lett. 85, 1004 (2000).

[6] T. I. C. Jansen and J. G. Snijders, J. Chem. Phys. 113, 307 (2000).

[7] S. Saito and I. Ohmine, Phys. Rev. Lett. 88, 207401 (2002).

[8] N. G. van Kampen, Phys. Norv. 5, 279 (1971).

[9] M. Kryvohuz and J. Cao (to be published).

[10] S. Mukamel, The Principles of Nonlinear Optical Spectroscopy (Oxford University, New York, 1995).

[11] S.R. de Groot and L.G. Suttorp, Foundations of Electrodynamics (Noord-Hollandsche U. M., Amsterdam, 1972).

[12] M. Hillery, R. F. O'Connel, M. O. Scully, and E. P. Wigner, Phys. Rep. 106, 121 (1984).

[13] E. J. Heller, J. Chem. Phys. 67, 3339 (1977).

[14] M. V. Berry, Phil. Trans. Roy. Soc. London, Ser. A 287, 237 (1977)

[15] C. Jaffe and P. Brumer, J. Chem. Phys. 82, 2330 (1985).

[16] C. Jaffe, S. Kanfer, and P. Brumer, Phys. Rev. Lett. 54, 8 (1985).

[17] M. L. Koszykowski, D. W. Noid, and R. A. Marcus, J. Phys. Chem. 86, 2113 (1982)

[18] C. W. Eaker, G. C. Schatz, N. D. Leon, and E. J. Heller, J. Phys. Chem. 83, 2990 (1985).

[19] W. H. Miller, Adv. Chem. Phys. 25, 69 (1974).

[20] W. H. Miller and A. W. Raczkowski, Faraday Discuss. Chem. Soc. 55, 45 (1973).

[21] M. V. Berry, in Chaos and Quantum Physics, edited by M. J. Giannoni, A. Voros, and J. Zinn- Justin (NorthHolland, Amsterdam, 1991).

[22] W. Heisenberg, The Physical Principles of the Quantum Theory (Dover, New York, 1949).

[23] W. R. Greenberg, A. Klein, and C.-T. Li, Phys. Rev. Lett. 75, 1244 (1995).

[24] M. Kryvohuz and J. Cao, J. Chem. Phys. 122, 024109 (2005).

[25] W. G. Noid and R. F. Loring, J. Chem. Phys. 122, 174507 (2005).

[26] P. Shah and C. Chakravartya, J. Chem. Phys. 116, 10825 (2002).

[27] C. Dellago and S. Mukamel, Phys. Rev. E 67, 035205(R) (2003). 